\title{
Beam induced hydrodynamic tunneling in the future circular collider components
}

\author{
N. A. Tahir, ${ }^{1}$ F. Burkart, ${ }^{2}$ R. Schmidt, ${ }^{2}$ A. Shutov, ${ }^{3}$ D. Wollmann, ${ }^{2}$ and A. R. Piriz ${ }^{4}$ \\ ${ }^{1}$ GSI Helmholtzzentrum für Schwerionenforschung, Planckstraße 1, 64291 Darmstadt, Germany \\ ${ }^{2}$ CERN, 1211 Geneva 23, Switzerland \\ ${ }^{3}$ Institute of Problems of Chemical Physics, Russian Academy of Sciences, \\ Institutskii pr. 18, 142432 Chernogolovka, Russia \\ ${ }^{4}$ E.T.S.I. Industriales, Universidad de Castilla-La Mancha, 13071 Ciudad Real, Spain
}

(Received 29 April 2016; published 3 August 2016)

\begin{abstract}
A future circular collider (FCC) has been proposed as a post-Large Hadron Collider accelerator, to explore particle physics in unprecedented energy ranges. The FCC is a circular collider in a tunnel with a circumference of $80-100 \mathrm{~km}$. The FCC study puts an emphasis on proton-proton high-energy and electron-positron high-intensity frontier machines. A proton-electron interaction scenario is also examined. According to the nominal FCC parameters, each of the $50 \mathrm{TeV}$ proton beams will carry an amount of 8.5 GJ energy that is equivalent to the kinetic energy of an Airbus A380 (560 t) at a typical speed of $850 \mathrm{~km} / \mathrm{h}$. Safety of operation with such extremely energetic beams is an important issue, as off-nominal beam loss can cause serious damage to the accelerator and detector components with a severe impact on the accelerator environment. In order to estimate the consequences of an accident with the full beam accidently deflected into equipment, we have carried out numerical simulations of interaction of a FCC beam with a solid copper target using an energy-deposition code (FLUKA) and a 2D hydrodynamic code (BIG2) iteratively. These simulations show that, although the penetration length of a single FCC proton and its shower in solid copper is about $1.5 \mathrm{~m}$, the full FCC beam will penetrate up to about $350 \mathrm{~m}$ into the target because of the "hydrodynamic tunneling." These simulations also show that a significant part of the target is converted into high-energy-density matter. We also discuss this interesting aspect of this study.
\end{abstract}

DOI: 10.1103/PhysRevAccelBeams.19.081002

\section{INTRODUCTION}

After the unprecedented success with the Large Hadron Collider (LHC), CERN launched a conceptual design study for a post-LHC particle accelerator, the future circular collider (FCC). The FCC is a circular collider with a circumference of $80-100 \mathrm{~km}$. Such an accelerator would allow particle physicists to push back the boundaries of knowledge even further; proton collisions are capable of reaching unprecedented energies in the range of $100 \mathrm{TeV}$. The study also includes an option for an electron-positron $\left(e^{+} e^{-}\right)$collider to be installed in the FCC tunnel before the proton-proton $(p p)$ collider; the $e^{+} e^{-}$collider could be used as a Higgs factory.

A conceptual design report will be delivered before the end of 2018, in time for the next update of the European Strategy for Particle Physics. The FCC study will be a global venture for particle physics and stems from the recommendation in the European Strategy for Particle Physics, published in May 2013, that a feasibility study

Published by the American Physical Society under the terms of the Creative Commons Attribution 3.0 License. Further distribution of this work must maintain attribution to the author(s) and the published article's title, journal citation, and DOI. be conducted on future fundamental research projects at CERN. The energy stored in each of the FCC proton beams would be on the order of $8.5 \mathrm{GJ}, 20$ times higher than the LHC and equivalent to the kinetic energy of an Airbus A380 (560 t) at a nominal speed $(850 \mathrm{~km} / \mathrm{h})$.

Already at the LHC the beam-stored energy increased by a factor of 100 with respect to previous machines such as TEVATRON, HERA, and RHIC. Therefore, a sophisticated, highly reliable machine protection system was designed and implemented in the LHC. The protection relies on a large number of sensors monitoring the correct functioning of the very complex hardware systems as well as from beam instruments for detecting any off-nominal beam parameters. At the end of a physics fill or in case of a failure, the beams are safely deposited by extracting them into $700 \mathrm{~m}$ long transfer lines, diluting the density, and finally absorbing the beam energy in large graphite blocks. The extraction system was designed to have a very high reliability; however, there are failure modes that could lead to the beam deflection with non-nominal angles, into a $10 \mathrm{~m}$ long graphite absorber, into a septum magnet, or into superconducting magnets. In order to estimate the risk, the consequences for such failures were estimated. One of the worst-case failures is an accidental deflection of the entire beam by a wrong angle straight into a vacuum chamber and magnet. There are several failure 
scenarios that can result in such an accident, for example, (i) failure in the energy-tracking system for kicker and septum magnets, (ii) firing of one or more, but not all, kicker magnets, and (iii) undetected failure of one of the septum magnets (such as an interturn short).

Although it is very unlikely that the entire beam is dumped on a spot, nevertheless, the consequences for such rare accidents should also be estimated.

For the LHC, the consequences of such a failure when operating with beams storing $362 \mathrm{MJ}$ were addressed by simulation studies of the impact of a LHC beam on a target. First, the energy deposition of the first bunches in a target is calculated with FLUKA [1,2], a fully integrated particle physics Monte Carlo simulation package, assuming solid material density. The effect of the energy deposition on the target is then calculated with a hydrodynamic code, BIG2 [3]. The impact of only a few tens of bunches leads to a significant change of target density at and around the beam axis. The calculations were done iteratively in several steps that showed that the LHC beam can penetrate up to 30-35 m into a copper target [4-7].

Experiments were performed at the CERN Super Proton Synchrotron (SPS), since simulation studies with the tools used for the LHC also predict hydrodynamic tunneling for the SPS beams [7]. An experiment at the SPS-HiRadMat facility (high radiation to materials) using the $440 \mathrm{GeV}$ beam with 144 bunches was performed in July 2012 [8,9]. The results of this experiment with the calculations of hydrodynamic tunneling were in very good agreement [10].

The protection systems for the FCC will be similar; in case of a failure that risks a result in beam losses, the beams need to be extracted from the machine and safely deposited. The extraction systems will be similar to those at LHC, with similar failure cases. Therefore, the consequences of an accidental deflection of the beam into the magnet system need to be studied. These calculations are documented in this paper.

It is important to emphasize that this problem involves beam-matter interaction studies, which is a very important and interesting field of research with wide applications to basic and applied physics, for example, ion-beam-driven inertial fusion [11-14] and the generation of high-energydensity (HED) matter [15-25]. Furthermore, development of the production targets for the generation of rare radioactive isotopes [26-29] as well as designing of targets for the stripping of energetic ions [30-32] also belong to this area of research.

In Sec. II, we present the nominal beam parameters for the FCC proton machine, while the problem of beammatter interaction and the phenomenon of hydrodynamic tunneling are described in Sec. III. A summary of the physics implemented in the FLUKA and the BIG2 codes is given in Sec. IV, whereas the numerical simulation results, with a detailed physical interpretation, are presented in Sec. V. Conclusions drawn from this work are noted in Sec. VI.

\section{NOMINAL BEAM PARAMETERS OF THE PROTON-PROTON FCC}

Each proton beam has the following characteristics: (i) particle energy, $50 \mathrm{TeV}$; (ii) total number of bunches, 10 600; (iii) bunch intensity, $10^{11}$ protons; (iv) number of protons per beam, $1.06 \times 10^{15}$; (v) bunch length, $0.5 \mathrm{~ns}$; (vi) bunch separation, $25 \mathrm{~ns}$; (vii) beam duration during impact on the target, $265 \mu \mathrm{s}$; (viii) transverse intensity distribution, Gaussian, characterized by $\sigma=0.2 \mathrm{~mm}$; and (ix) total energy per beam, 8.5 GJ.

\section{BEAM-MATTER INTERACTION AND HYDRODYNAMIC TUNNELING}

The FCC beam parameters presented in Sec. II translate into an energy of $800 \mathrm{~kJ}$ per bunch. Numerical simulations have shown that the energy deposited in the target by a single FCC bunch strongly heats the target material and generates extremely high pressures. The high pressure in the absorption region drives a strong radially outgoing shock wave that leads to a substantial density depletion at and around the beam axis. As a consequence, the protons that are delivered in the subsequent bunches, and the hadronic shower they generate, penetrate deeper into the target. Continuation of this process leads to a substantial increase in the range of the projectile particles and the shower. This phenomenon is called "hydrodynamic tunneling" of ultrarelativistic particle beams. This was originally introduced and simulated for the $20 \mathrm{TeV}$ superconducting supercollider beam [33,34].

Similar calculations have previously been done using the $7 \mathrm{TeV}$ proton LHC beam impinging on a solid copper cylindrical target. The static range of a single $7 \mathrm{TeV}$ proton and its shower in solid copper is about $1 \mathrm{~m}$. However, when a full hydrodynamic calculation is done using the entire beam (duration $89 \mu \mathrm{s}$ ), the penetration depth becomes about $35 \mathrm{~m}$ [6]. Because of the implications of this phenomenon on machine protection design, it was considered necessary to have experimental verification of this effect. It was decided at CERN to perform beam-target heating experiments at the HiRadMat facility using the $440 \mathrm{GeV}$ proton beam delivered by the SPS in order to check the validity of the theoretical calculations [8,9]. Detailed numerical simulations were also carried out using the beam parameters that were used in the experiments [10]. These simulations were done employing the energydeposition code FLUKA $[1,2]$ and a 2D hydrodynamic code (BIG2 [3]), iteratively. This means that first the FLUKA code is run to calculate the energy-deposition distribution of the $440 \mathrm{GeV}$ protons considering the solid target density. These data are used as input to BIG2, which calculates the thermodynamic and the hydrodynamic processes in the target until the density along the target axis in the beamheated region is depleted by about $15 \%$. The code is then stopped, the modified density distribution provided by BIG 2 


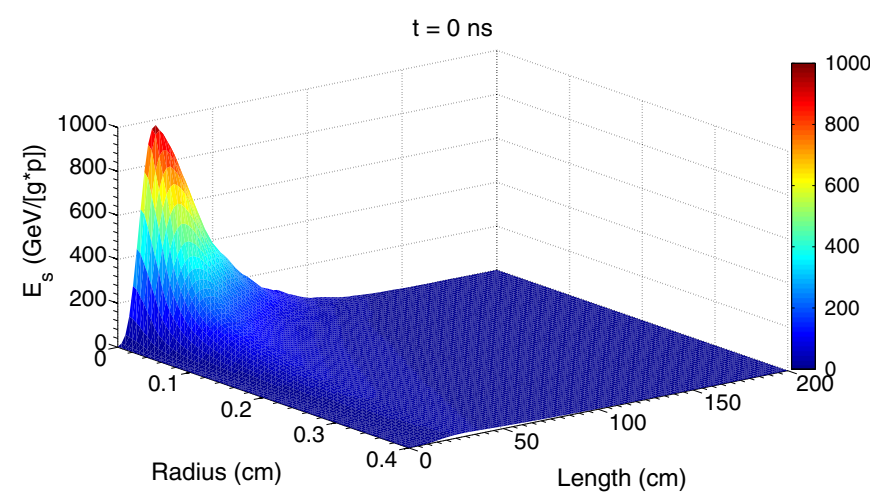

(a)

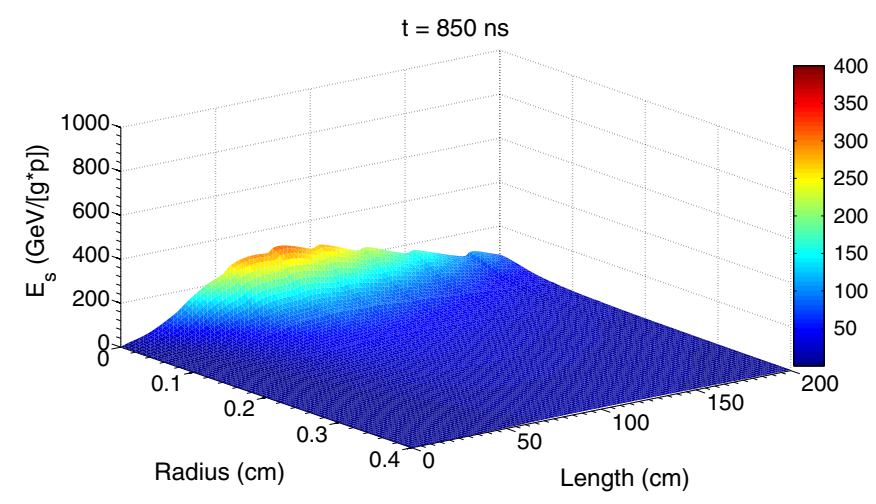

(c)

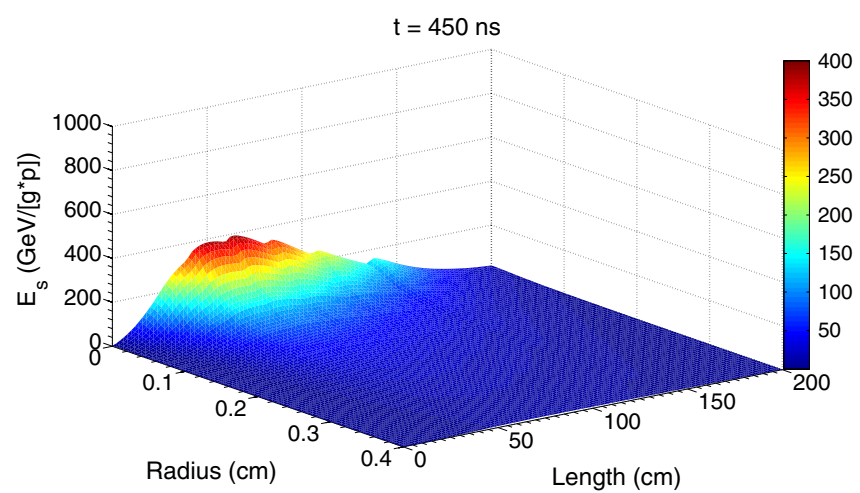

(b)

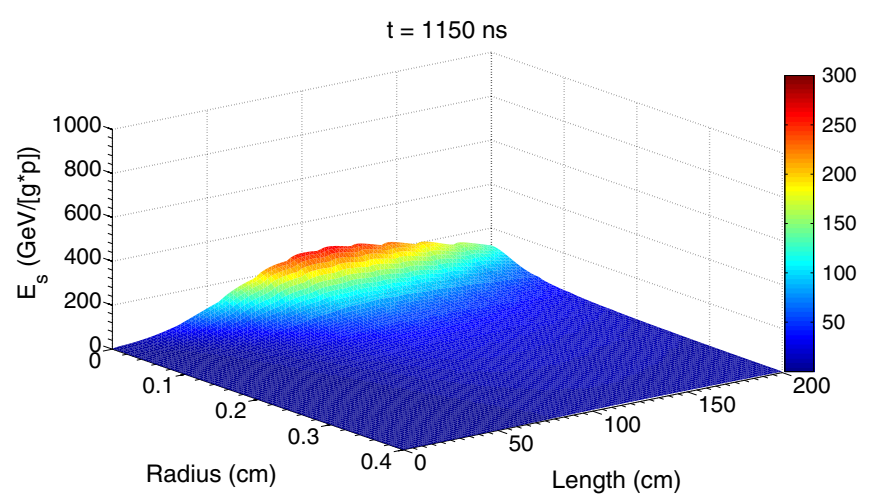

(d)

FIG. 1. Specific energy deposition $E_{s}$ for a $40 \mathrm{TeV}$ FCC proton in a solid copper cylinder having radius $r=2 \mathrm{~cm}$, length $L=500 \mathrm{~cm}$, with facial irradiation, beam spot size characterized with standard deviation $\sigma=0.2 \mathrm{~mm}$ using the FLUKA code (a) using a solid density of $8.93 \mathrm{~g} / \mathrm{cm}^{3}$; (b) using the density distribution provided by BIG2 at $t=450 \mathrm{~ns}$, (c) using the density distribution provided by BIG2 at $t=850 \mathrm{~ns}$, and (d) using the density distribution provided by BIG2 at $t=1150 \mathrm{~ns}$ (FLUKA calculates energy deposition in the target by the beam with a defined spot size; the result is then downscaled for a single proton to allow an easy scaling depending on the total intensity).

is used in the FLUKA code to calculate the energydeposition distribution for the next iteration, and the process continues until the last bunch hits the target. To have a good accuracy of the calculations, the energy-deposition distribution should not change too much from one iteration to the next. For this reason, we allow the peak value of the energydeposition distribution to vary by about $10 \%-15 \%$ between two successive iterations. An iterative step of $700 \mathrm{~ns}$ has been found suitable to fulfill this condition. Further details about the simulations can be found in Ref. [10].

\section{PHYSICS OF FLUKA AND BIg2 CODES}

FLUKA is a fully integrated particle physics and multipurpose Monte Carlo simulation package, capable of simulating all components of the particle cascades in matter up to $\mathrm{TeV}$ energies. FLUKA has many applications in highenergy experimental physics and engineering, shielding, detector and telescope design, cosmic ray studies, dosimetry, medical physics, and radiobiology, as well as allows one to simulate the interaction of beams with matter over a very wide energy range. The maximum energy of projectile particles can be as high as $10^{4} \mathrm{TeV}$ and minimum energy as low as $1 \mathrm{keV}$ for photons and electrons and a fraction of an electronvolt for neutrons.

More details about the used models and their performances, as well as a vast amount of benchmarking, can be found in Refs. [1,2]. It is to be noted that the models used in FLUKA also include a nuclear-size correction to the stopping power at very high energies.

BIG2 is a two-dimensional hydrodynamic computer code based on a Godunov-type numerical scheme. It uses a stable and versatile numerical mesh that can handle simple as well as complicated geometries of single- and multilayered targets. A heat conductivity package and an ion beam energy-deposition module are also included. In the case of the SPS, LHC, and FCC proton beams, energydeposition data provided by the FLUKA code are taken, whereas, for the heavy ions, the energy-deposition data calculated by the SRIM code [35] are used as energy input. 


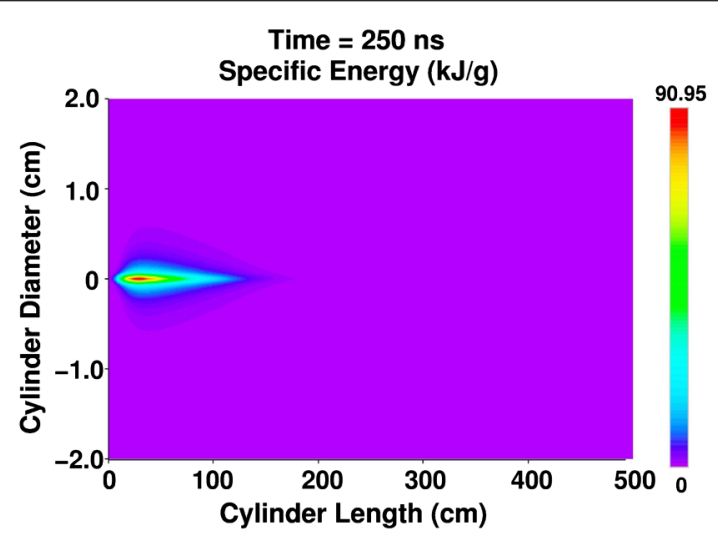

(a)

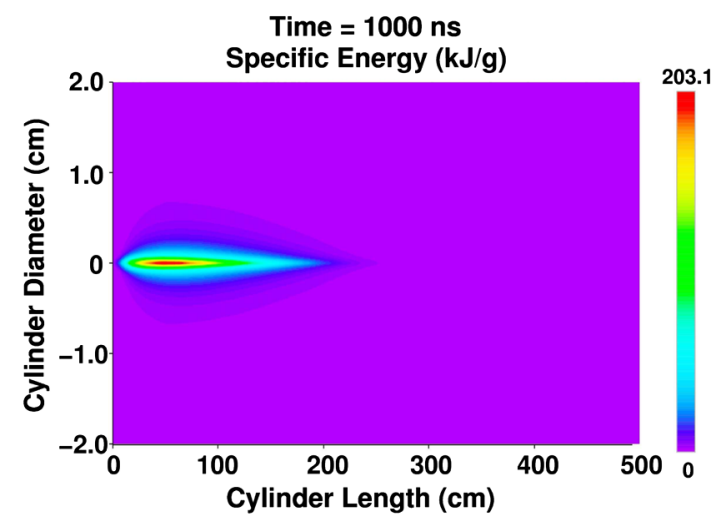

(c)

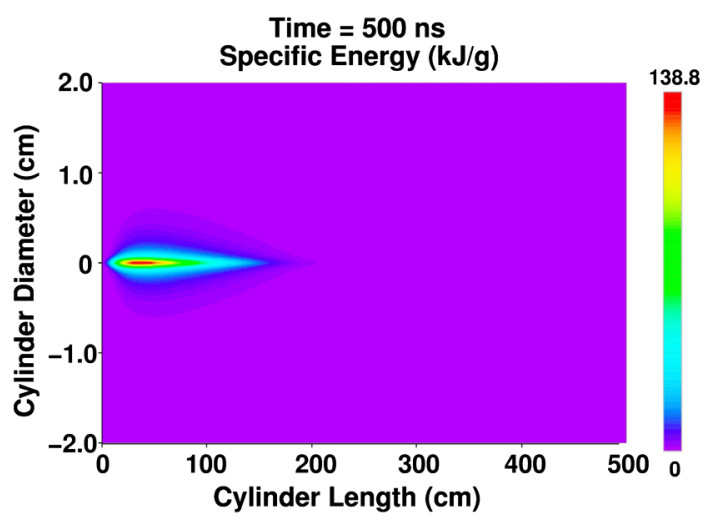

(b)

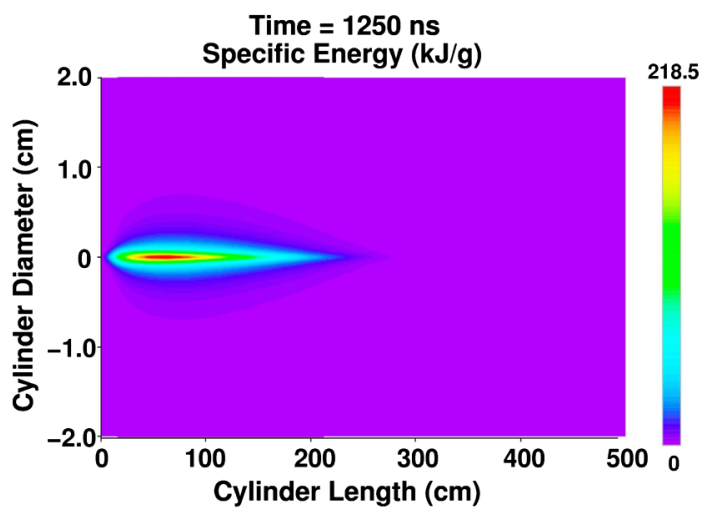

(d)

FIG. 2. Specific energy deposition in the target calculated by BIG2 (a) at $t=250 \mathrm{~ns}$ (10 bunches delivered), (b) at $t=500 \mathrm{~ns}$ (20 bunches delivered), (c) at $t=1000 \mathrm{~ns}$ (40 bunches delivered), and (d) at $t=1250 \mathrm{~ns}$ (50 bunches delivered).

The elastic plastic effects are treated assuming an ideal plasticity model based on Hooks law complemented with von Mises yield criterion. Different phases of the target material during and after the irradiation are treated using a semiempirical equation-of-state (EOS) model [36,37].

\section{NUMERICAL SIMULATION RESULTS AND THEORETICAL ANALYSIS}

In this section, we present the numerical simulation results of the impact of a FCC beam using nominal beam parameters given in Sec. II. However, it is to be noted that, when this work was started, the FCC beam parameters were not yet fixed. At that time, $40 \mathrm{TeV}$ was considered to be a reasonable particle energy for the purpose of such calculations. These calculations are, therefore, done assuming $40 \mathrm{TeV}$ proton energy, but in principle, these results can easily be extrapolated to $50 \mathrm{TeV}$ protons.

\section{A. Energy-deposition simulations using FLUKA code}

The energy deposition by a proton beam with a defined size is obtained with FLUKA by simulating the impact of a large number of protons representing the beam on a target. This yields the energy deposition of the beam, and, from the results, the energy deposition for a single proton is obtained. The energy deposition of a single bunch is then calculated by multiplying the number of protons in a bunch with this result. For these calculations, the considered target is a solid copper cylinder (density $8.93 \mathrm{~g} / \mathrm{cm}^{3}$ ) having a radius of $2 \mathrm{~cm}$ and a length of $5 \mathrm{~m}$ that is facially irradiated. The results are presented in Fig. 1(a), where specific energy deposition is plotted on a diameter-length plane. It is seen that the peak specific energy deposition is $950 \mathrm{GeV} / \mathrm{g}$, and it lies along the axis at a position $L=23 \mathrm{~cm}$.

As explained in Sec. III, these data are used as input to the BIG2 code, and the code is allowed to calculate the thermodynamic and the hydrodynamic response of the target material. The hydrodynamic processes lead to a density depletion in the absorption region, and, when the density reduction becomes of the order of $15 \%$, the BIG2 calculations are stopped for the next iteration of energydeposition calculations.

It is important to note that the first FCC bunch with $40 \mathrm{TeV}$ energy induces about $14 \mathrm{~kJ} / \mathrm{g}$ specific energy in solid copper that is sufficient to evaporate the material and generates a thermal pressure of about 1 Mbar. The hydrodynamic effects produced by this pressure are strong enough to reduce the axial target density by $15 \%$ within $25 \mathrm{~ns}$ (before the second bunch arrives). Therefore, to ensure good accuracy of the calculations, we use an 
iteration step of $25 \mathrm{~ns}$ during the initial stages of the calculations which makes the calculations very time consuming. At a later stage, however, when the hydrodynamic processes become slower, the iteration time is steadily increased to $100 \mathrm{~ns}$.

To show the influence of changes in the target density distribution on the energy-deposition calculations, we plot in Fig. 1(b) the FLUKA energy-deposition distribution corresponding to the density distribution provided by the BIG2 code at $t=450 \mathrm{~ns}$, when 18 FCC bunches have been delivered. It is seen that the peak has been significantly broadened and the maximum value has been reduced to about $400 \mathrm{GeV} / \mathrm{g}$. The former effect is due to the deeper penetration of the beam, while the latter effect is due to the density reduction. These effects are much more pronounced in Figs. 1(c) and 1(d), where we present the FLUKA energydeposition distribution using the density distributions provided by the BIG2 code at $t=850$ and $1150 \mathrm{~ns}$, respectively. It is seen in Fig. 1(c) that the peak has been reduced to $300 \mathrm{GeV} / \mathrm{g}$, while in Fig. 1(d) this value is around $250 \mathrm{GeV} / \mathrm{g}$. The energy-deposition distribution is very much broadened in the two cases, and a deeper penetration of the hadronic shower is clearly seen.

It is to be noted that the surface of the energy-deposition distribution in Fig. 1(a) is smooth (solid density case), whereas in the remaining three figures, it shows a nonsmooth (kink-shaped) behavior. This is because the first FCC bunch deposits a large specific energy that produces a high pressure of around $1 \mathrm{Mbar}$ in the absorption region. This high pressure generates a two-dimensional shock wave that compresses the material in the radial as well as in the longitudinal direction. The shock is very strong in the radial direction, and it leads to substantial density depletion at and around the axis before the arrival of the second bunch. As the second and the subsequent bunches penetrate the target, after traveling through the low-density region, they encounter the supersolid density region generated by the longitudinal shock propagation. This leads to a higher energy deposition in that zone which generates a kink-shaped behavior in the energy-deposition surface in the latter figures.

\section{B. Thermodynamic and hydrodynamic simulation results using BIG2 code}

Next, we present the thermodynamic and the hydrodynamic part of our calculations that have been done employing the 2D hydrodynamic code BIG2. The energydeposition distributions calculated with the FLUKA code are used in BIG2 as an input, and the calculations are done using an iterative scheme as explained above.

\section{Specific energy deposition}

The simulation results of specific energy deposited by the FCC proton bunches and their hadronic shower in the

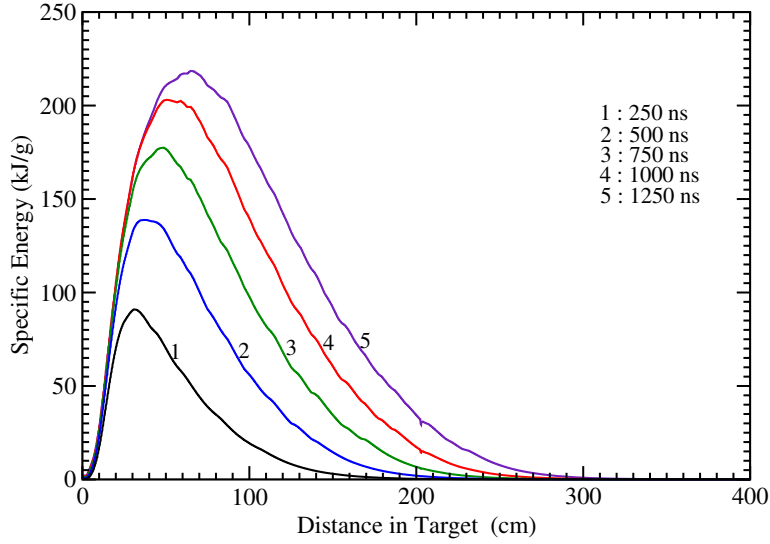

(a)

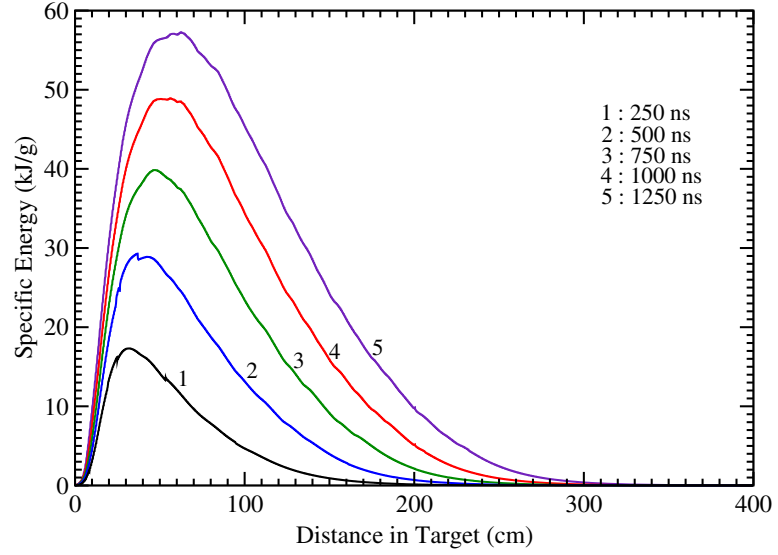

(b)

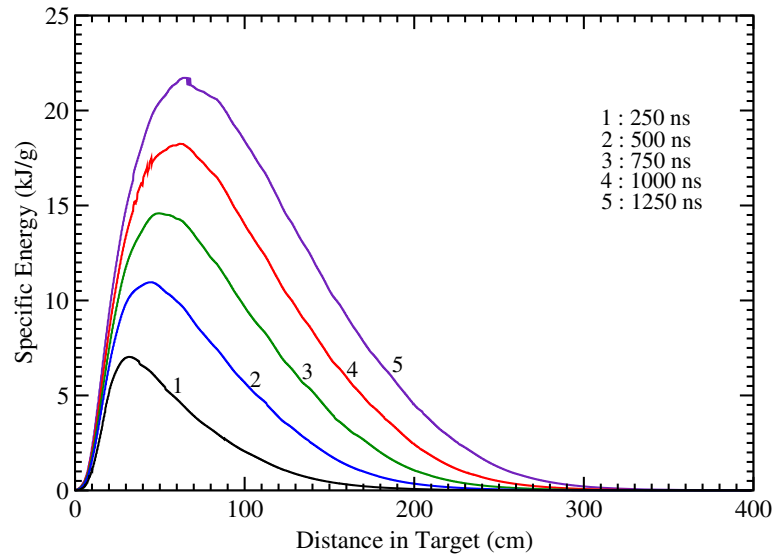

(c)

FIG. 3. Specific energy-deposition profiles vs distance in the target at different times: (a) along the axis at $r=0$, (b) at $r=1 \mathrm{~mm}$, and (c) at $r=2 \mathrm{~mm}$.

target are shown, using two-dimensional as well as onedimensional graphics, obtained by the BIG2 calculations. First, we plot in Fig. 2 the specific energy-deposition distribution in the target on a diameter-length plane.

Figure 2(a) shows that at $t=250 \mathrm{~ns}$, when ten bunches have been delivered, a maximum specific energy of about $90 \mathrm{~kJ} / \mathrm{g}$ is deposited and the shower penetration length is around $1.7 \mathrm{~m}$. 


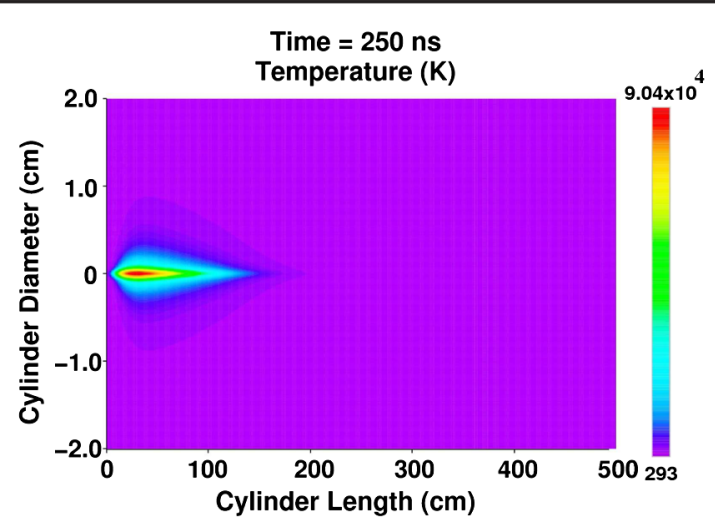

(a)

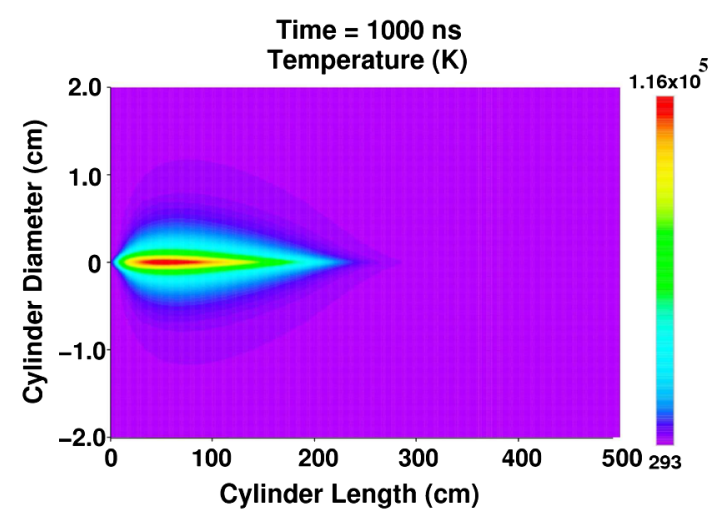

(c)

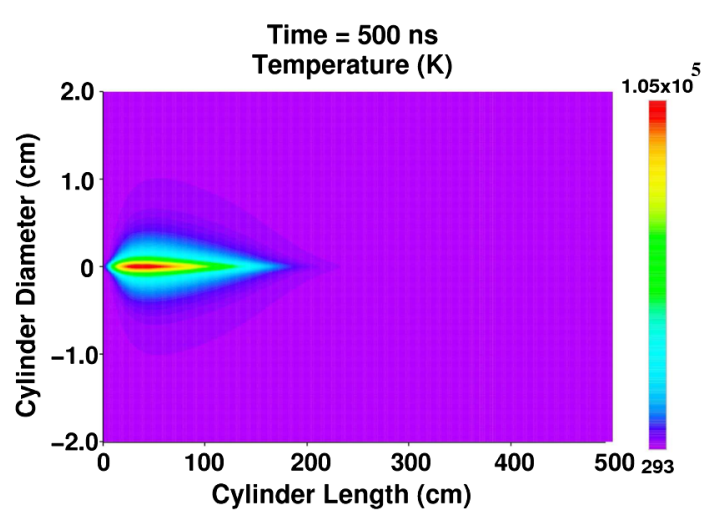

(b)

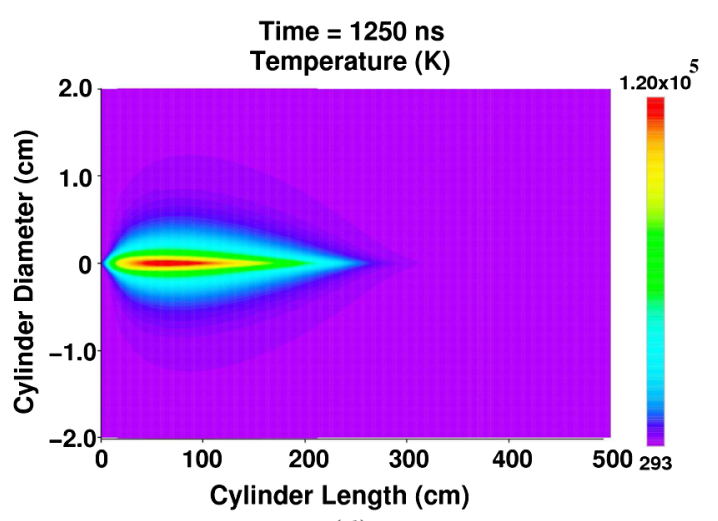

(d)

FIG. 4. Temperature distribution in the target calculated by BIG2 (a) at $t=250 \mathrm{~ns}$ (10 bunches delivered), (b) at $t=500 \mathrm{~ns}$ (20 bunches delivered), (c) at $t=1000 \mathrm{~ns}$ (40 bunches delivered), and (d) at $t=1250 \mathrm{~ns}$ (50 bunches delivered).

Figure 2(b) is plotted at $t=500 \mathrm{~ns}$, which is the time when 20 bunches have been delivered. It is seen that the maximum value of the specific energy deposition is of the order of $140 \mathrm{~kJ} / \mathrm{g}$ and the penetration length of the hadronic shower is about $2.2 \mathrm{~m}$.

Figure 2(c) shows that the maximum value of the specific energy at $t=1000 \mathrm{~ns}$ (40 bunches delivered) has increased to around $200 \mathrm{~kJ} / \mathrm{g}$, while the penetration depth of the shower becomes around $2.8 \mathrm{~m}$.

In Fig. 2(d), we present the specific energy deposition at $1250 \mathrm{~ns}$ (50 bunches delivered). It is seen that the maximum specific energy deposition is $218.5 \mathrm{~kJ} / \mathrm{g}$ and the shower has penetrated up to about $3 \mathrm{~m}$ into the target along the axis.

It is important to note that the maximum specific energy deposition does not increase linearly with the bunch number, but the rate of increase slows down with time. This is because, due to the hydrodynamic tunneling, the heated volume continuously increases that leads to the distribution of the energy of the subsequent bunches over an ever-increasing material mass. This is clearly seen in Figs. 2(a)-2(d) as the dimension (volume) of the beamheated region continuously increases with time.

One-dimensional profiles of the specific energy deposition calculated with BIG2 are presented in Fig. 3. In
Fig. 3(a), we show the specific energy deposition along the axis $(r=0)$ at different times during irradiation. It is seen that, due to the continuous delivery of the bunches to the target, the specific energy deposition increases with time, but the rate of increase of the maxima of the curve slows down with time due to the reasons mentioned above. Moreover, deeper penetration of the protons and the shower as a function of time are clearly seen.

Figure 3(b) shows the same parameters as Fig. 3(a), but at a radial position $r=1 \mathrm{~mm}$. It is seen that, even at this distance from the axis, a large amount of energy is deposited that is sufficient to evaporate the material. It is interesting to note that the curves show that the maximum value of the deposited energy continues to increase at a faster rate, as compared to that in Fig. 3(a). This is because the material density at this radial position is significantly higher than that at the axial position.

In Fig. 3(c) are plotted the specific energy-deposition curves along the cylinder length at a radial position $r=2 \mathrm{~mm}$, at different times. It is seen that, even at this relatively large distance from the beam axis, enough energy is deposited that can severely damage the material. However, due to a higher material density compared to the other two radial positions, the maximum value of the energy deposition still increases at a steady rate. 


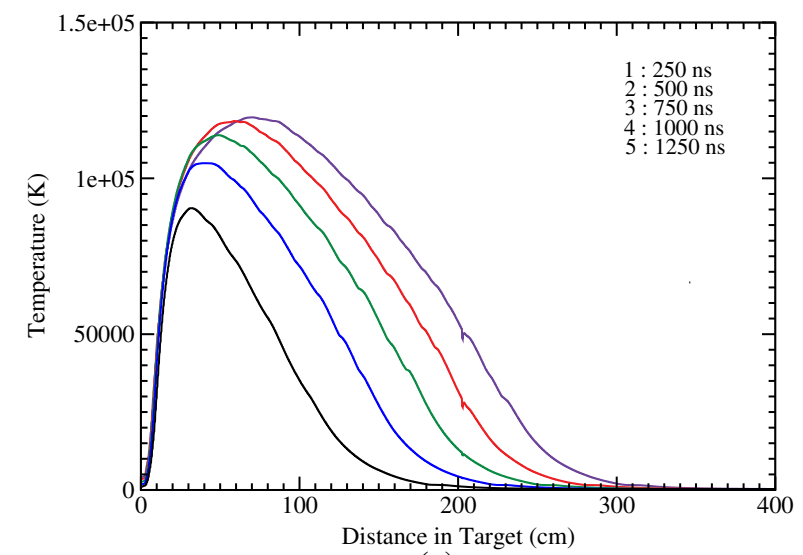

(a)

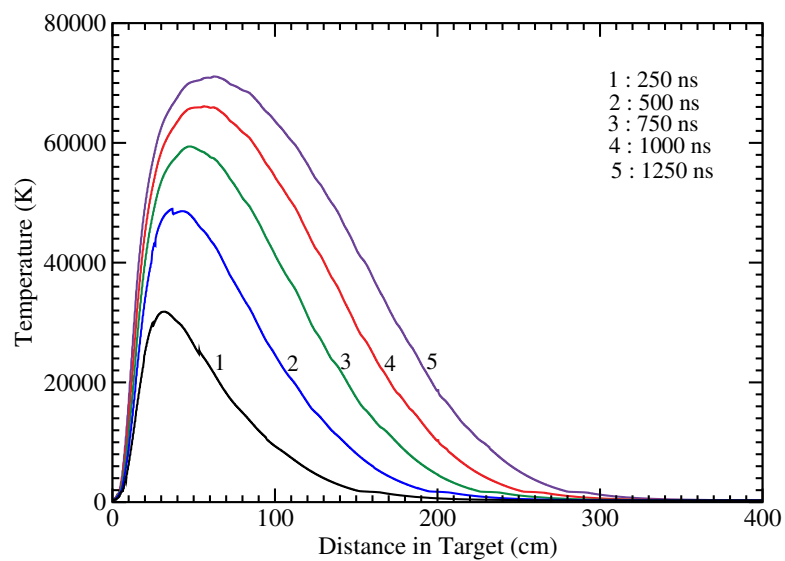

(b)

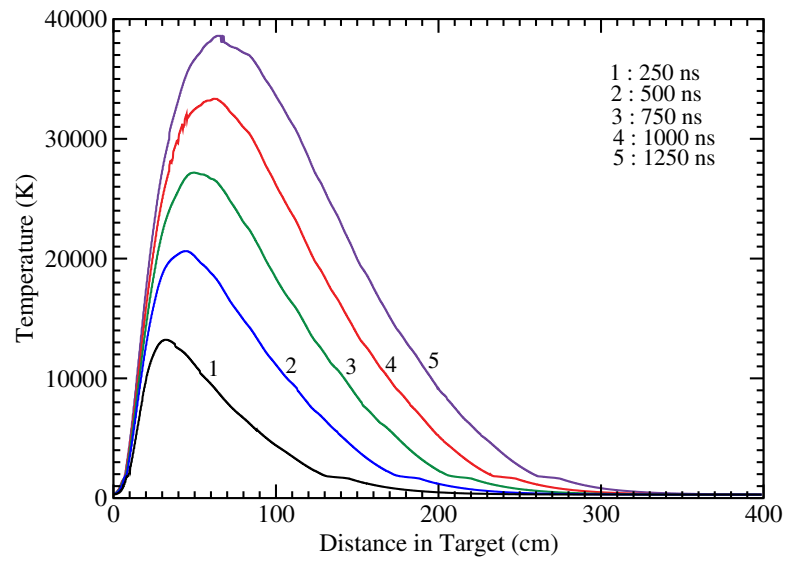

(c)

FIG. 5. Target temperature profiles vs distance in the target at different times: (a) along the axis at $r=0$, (b) at $r=1 \mathrm{~mm}$ and (c) $r=2 \mathrm{~mm}$.

\section{Target temperature}

The high level of energy deposition in the target material causes strong heating that leads to very high temperatures. In Figs. 4(a)-4(d), we present the temperature field generated in the target (calculated by BIG2) corresponding to the specific energy deposition plotted in Figs. 2(a)-2(d), respectively.

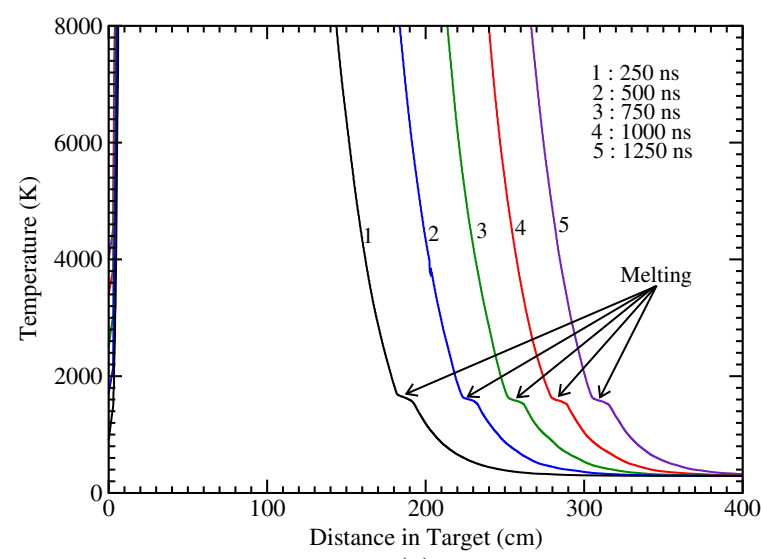

(a)

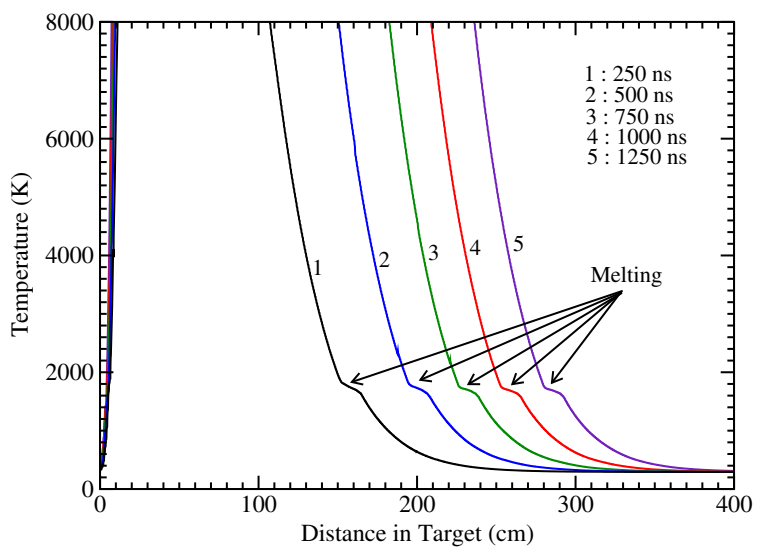

(b)

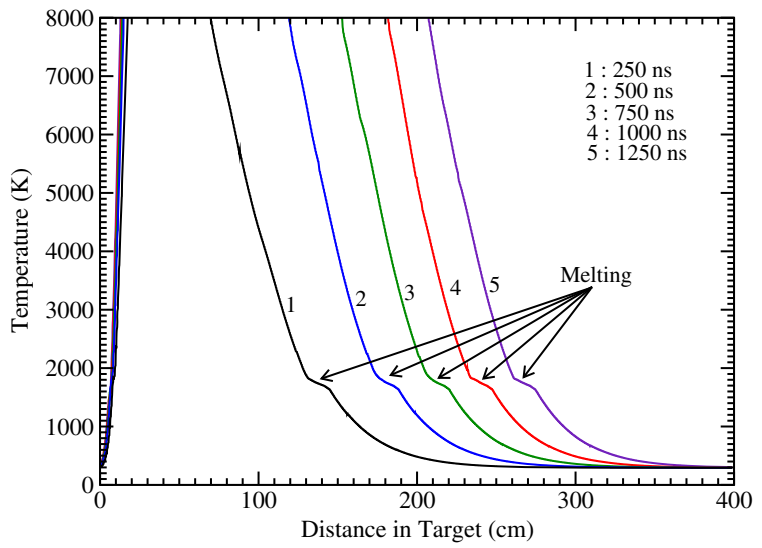

(c)

FIG. 6. Magnified view of the temperature profiles presented in Fig. 5.

Figure 4(a) shows that, at $t=250 \mathrm{~ns}$, a maximum temperature of $9 \times 10^{4} \mathrm{~K}$ is generated. This means that, after the delivery of just ten FCC bunches, the beam-heated region will be seriously damaged.

Figure 4(b) is plotted at $t=500 \mathrm{~ns}$ that shows a maximum temperature of about $10^{5} \mathrm{~K}$, while the extension 


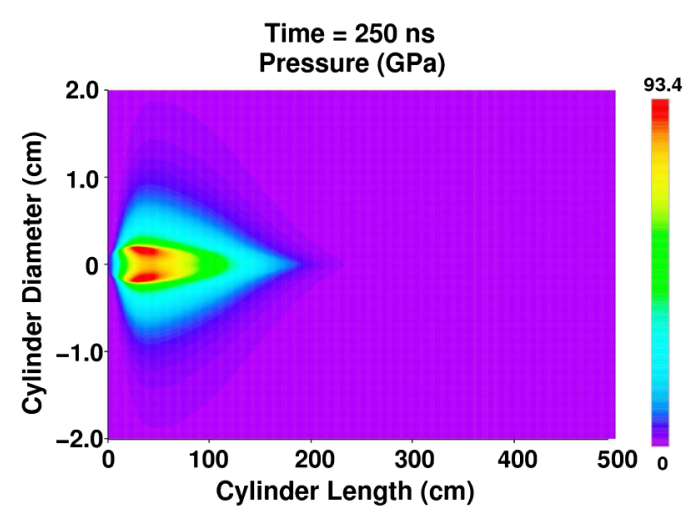

(a)

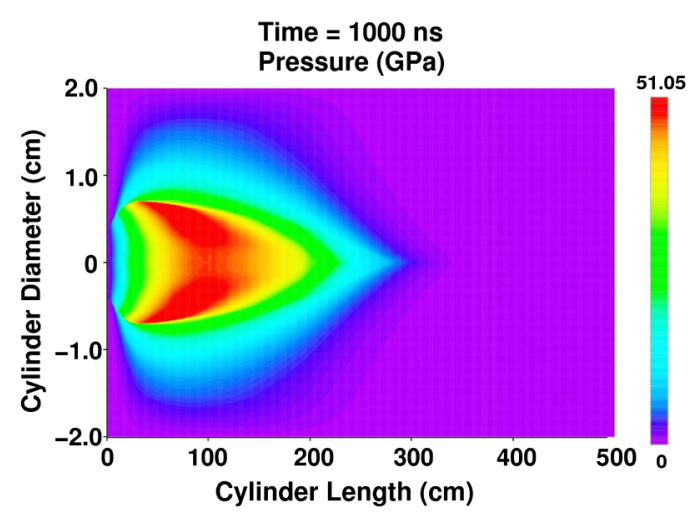

(c)

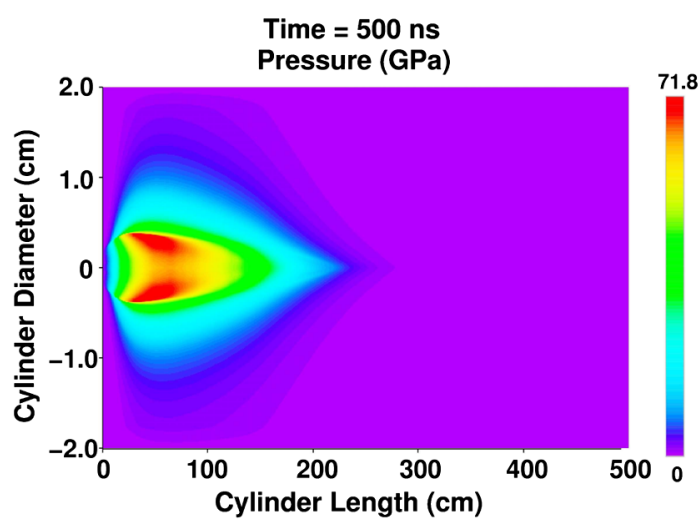

(b)

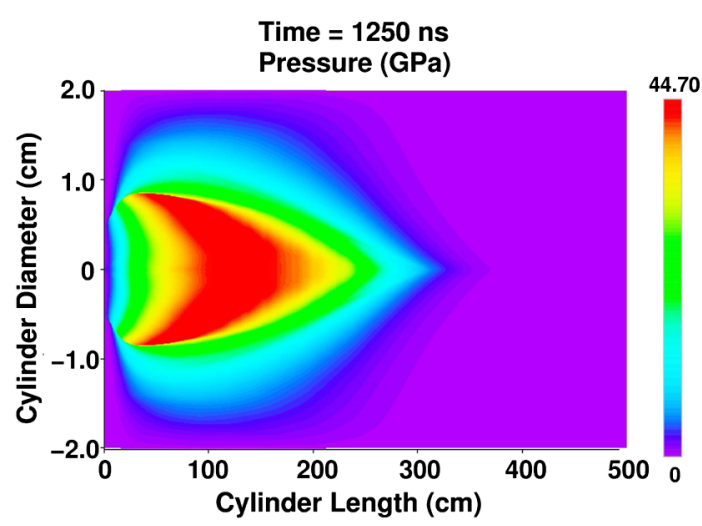

(d)

FIG. 7. Target pressure distribution calculated by BIG2 (a) at $t=250 \mathrm{~ns}$ (10 bunches delivered), (b) at $t=500 \mathrm{~ns}$ (20 bunches delivered), (c) at $t=1000 \mathrm{~ns}$ (40 bunches delivered), and (d) at $t=1250 \mathrm{~ns}$ (50 bunches delivered).

of the heated region, both in the radial as well as in the longitudinal direction, is clearly seen. Extension of the heating zone in the longitudinal direction is caused by the hydrodynamic tunneling effect, whereas, in the radial direction, it occurs due to the accumulative effect of the energy deposition by the shower generated by the subsequent proton bunches.

Figure 4(c) is plotted at $t=1000 \mathrm{~ns}$, when $40 \mathrm{FCC}$ bunches have deposited their energy in the target. It is seen that there is no significant increase in the maximum value of the temperature; however, the dimensions of the heated zone further increase in both the $r$ and $z$ directions. The same behavior is seen in Fig. 4(d), where the temperature field is presented at $t=1250 \mathrm{~ns}$, which corresponds to the time of delivery of 50 FCC bunches.

Next, we present in Figs. 5(a)-5(c) profiles of the temperature vs distance into the target at different radial positions including $r=0,1$, and $2 \mathrm{~mm}$, respectively. It is seen in Fig. 5(a) that the temperature along the axis steadily increases with time and the heating front moves deeper into the target due to the hydrodynamic tunneling. It is also interesting to note that the peak of the curve $5(t=1250 \mathrm{~ns})$ has shifted towards the right compared to that in curve 4 $(t=1000 \mathrm{~ns})$. This is because of the strong reduction in the target density in that region, in the case of curve 5.
Figure 5(b) shows that, at a radial position of $1 \mathrm{~mm}$, the maximum temperature increases to about $70000 \mathrm{~K}$ (curve 5) after the delivery of 50 FCC bunches. The corresponding curve in Fig. 5(c) shows that the temperature at the radial position of $2 \mathrm{~mm}$ becomes $40000 \mathrm{~K}$ due to the high level of energy deposition.

It is also important to study the material state at the boundary of the beam-heated and the cold material. For this purpose, we present in Figs. 6(a)-6(c) a magnified view of the temperature profiles vs distance into the target at different radial positions including $r=0,1$, and $2 \mathrm{~mm}$, respectively, at different times. These figures show that every temperature profile has a flat part which represents the melting of the target material at that location. It is also seen that this flat region (melting front) continuously shifts towards the right as the boundary of the beam-heated region extends in the beam direction due to the hydrodynamic tunneling. Figure 6(a) shows that, at the axis, the melting region lies between $L=300$ and $310 \mathrm{~cm}$ at $t=$ $1250 \mathrm{~ns}$ (delivery of $50 \mathrm{FCC}$ bunches). At radial positions 1 and $2 \mathrm{~mm}$, the corresponding melting zones are located between $L=280-290$ and $260-270 \mathrm{~cm}$, respectively. This is consistent with the Gaussian intensity distribution in the focal spot. This analysis shows that the target will not only be damaged at the axis, but melting and 


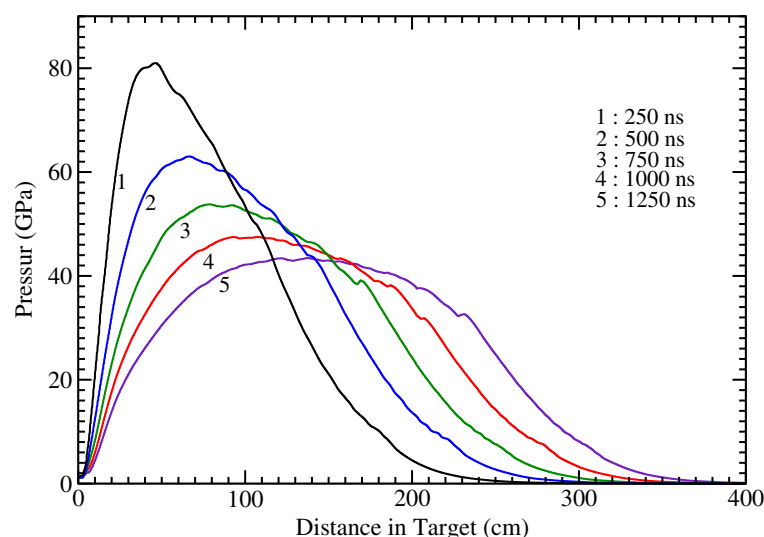

(a)

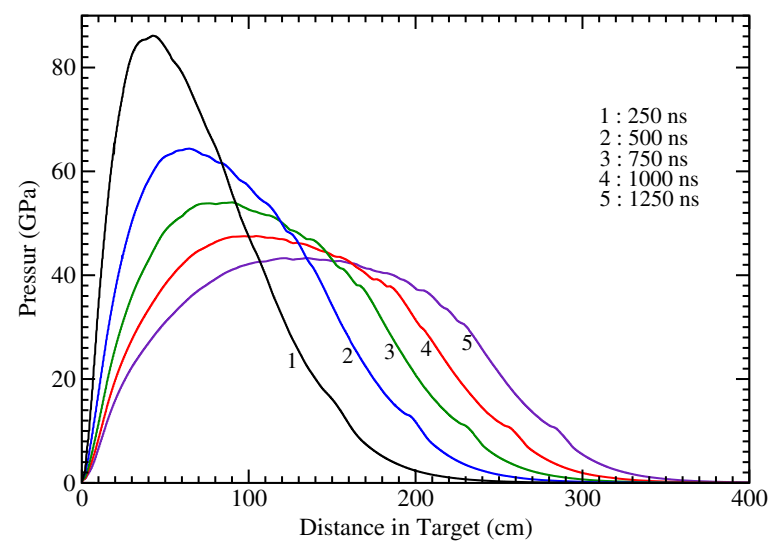

(b)

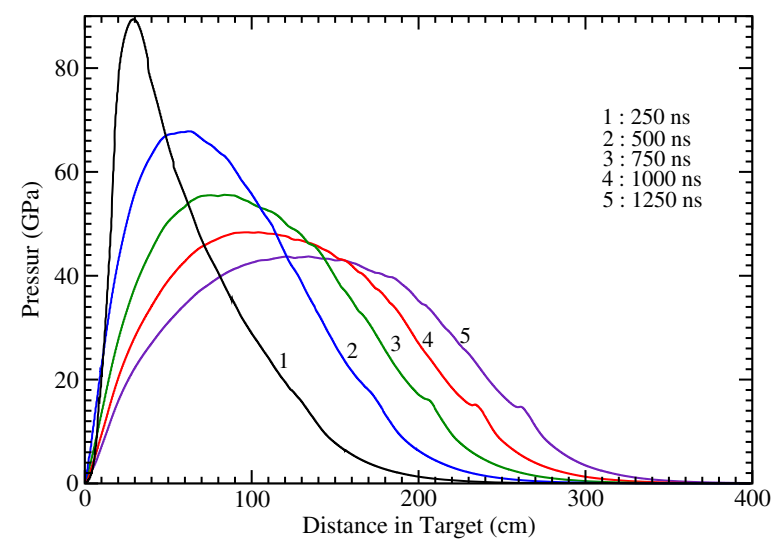

(c)

FIG. 8. Pressure profiles vs distance in the target at different times: (a) along the axis at $r=0$, (b) at $r=1 \mathrm{~mm}$, and (c) at $r=2 \mathrm{~mm}$.

evaporation of the material can occur in a large volume around the axis.

\section{Target pressure}

The high temperature generates very high pressure in the heated zone that drives a very strong outgoing radial shock wave, which leads to substantial modifications in the density distribution throughout the target. In order to analyze these effects, it is important to study the evolution of the pressure distribution at different times during the irradiation. It is worth noting that, initially, when the target is at a solid density, in addition to a strong radial shock wave, a weaker shock wave is also generated in the longitudinal direction. This leads to a two-dimensional shock front with varying density that influences the energy deposition. However, when the hydrodynamic tunneling sets in, the shock wave in the longitudinal direction is overtaken by the effects of deeper penetration of the protons and the shower, while, in the radial direction, the shock wave continues to propagate undisturbed, thereby strongly depleting the density at and around the axis.

In Fig. 7(a), we present the pressure distribution in the target at $t=250 \mathrm{~ns}$. It is seen that a maximum pressure of around $90 \mathrm{GPa}$ has been generated in the absorption region and an outgoing radial shock wave has already developed. Figure 7(b) shows the pressure distribution at $t=500 \mathrm{~ns}$. It is seen that the maximum value of the pressure has been reduced to about $70 \mathrm{GPa}$ because of the diverging cylindrical geometry and the shock front has moved further outwards. Figure 7(c) shows that, at $t=1000 \mathrm{~ns}$, the maximum value of the pressure has been reduced to around $50 \mathrm{GPa}$ and the shock has propagated further towards the cylinder surface. Similar behavior is seen in Fig. 7(d), at $1250 \mathrm{~ns}$.

The pressure profiles vs target length, at different times, at three different radial positions, namely, $r=0$ (axis), $r=1 \mathrm{~mm}$, and $r=2 \mathrm{~mm}$, are plotted in Figs. 8(a)-8(c), respectively. It is seen in Fig. 8(a) that, at $t=250 \mathrm{~ns}$, the peak pressure at the axis is about $80 \mathrm{GPa}$. At $500 \mathrm{~ns}$, the maximum pressure has been reduced to around $60 \mathrm{GPa}$ because of the density reduction caused by the radially outmoving shock wave. The following curves show a further reduction in the maximum pressure due to this effect, while the pressure profile becomes flatter with time. Moreover, the right boundary of the pressure curve continuously extends towards the right which is caused by the hydrodynamic tunneling.

The curves plotted in Figs. 8(b) and 8(c) show a similar behavior of the pressure evolution at radial positions 1 and $2 \mathrm{~mm}$, respectively.

\section{Target density}

The effect of the hydrodynamic processes on the target density is shown in Figs. 9(a)-9(d), where we present the density distribution on a diameter-length plane at different times including 250, 500, 1000, and $1250 \mathrm{ns,} \mathrm{respectively.}$ These figures show a qualitative view of the density evolution during the irradiation. More quantitative information is provided by the one-dimensional density profiles presented in Figs. 10(a)-10(c).

Figure 9(a) shows that at $t=250 \mathrm{~ns}$, which is the time when ten bunches have been delivered, the density at the 


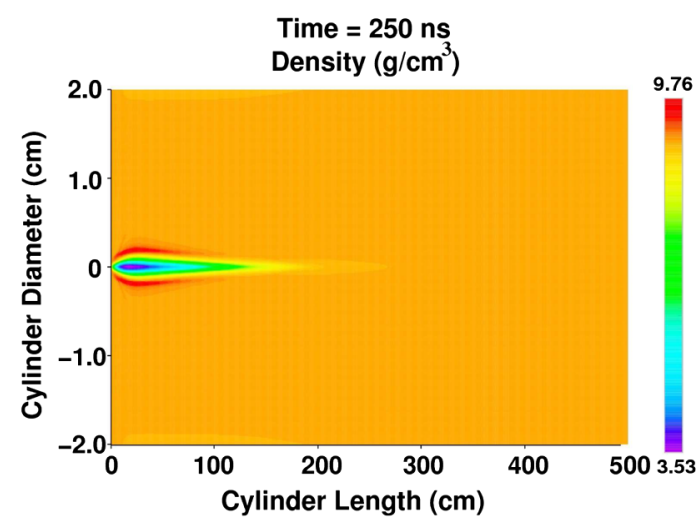

(a)

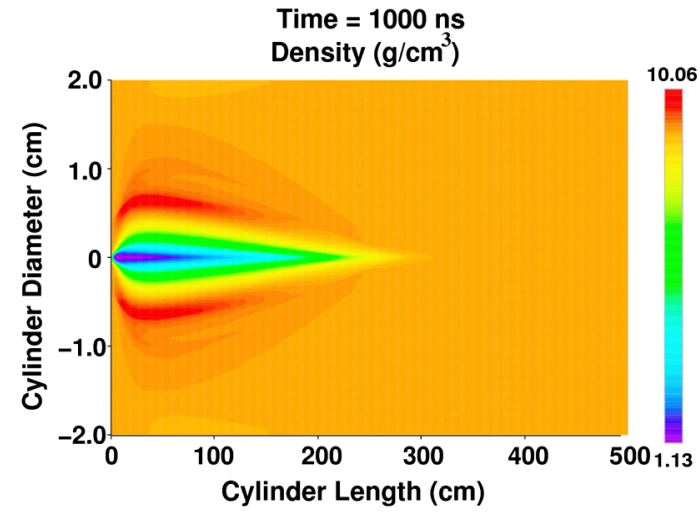

(c)

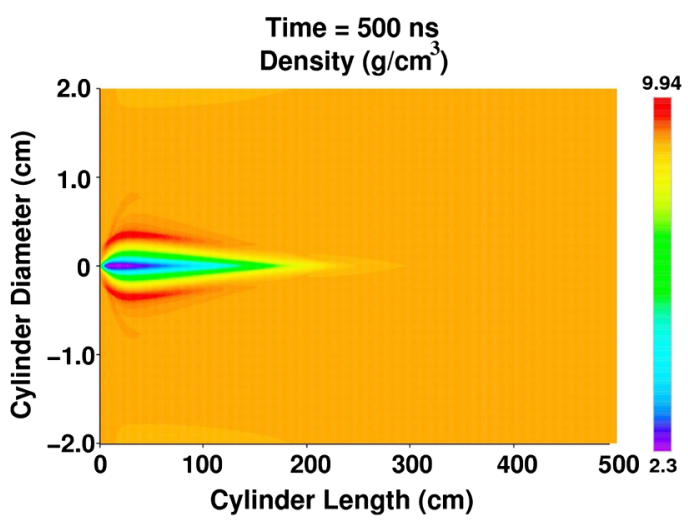

(b)

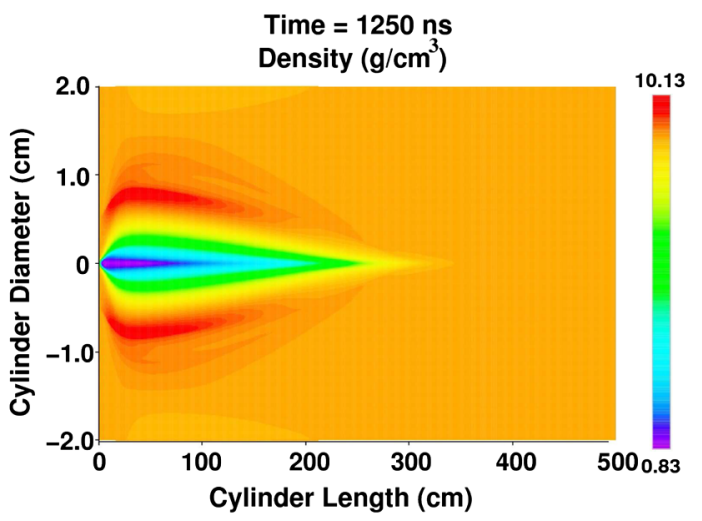

(d)

FIG. 9. Target density distribution calculated by BIG2 (a) at $t=250 \mathrm{~ns}$ (10 bunches delivered), (b) at $t=500 \mathrm{~ns}$ ( 20 bunches delivered), (c) at $t=1000 \mathrm{~ns}$ (40 bunches delivered), and (d) at $t=1250 \mathrm{~ns}$ (50 bunches delivered).

cylinder axis has been reduced to about $3.5 \mathrm{~g} / \mathrm{cm}^{3}$, while the radially outmoving shock front has a density of $9.78 \mathrm{~g} / \mathrm{cm}^{3}$ (see the maximum and the minimum value at the color scale).

Figure 9(b) presents the density distribution at $500 \mathrm{~ns}$, when 20 bunches have been delivered. The color scale on this figure shows that the minimum value of the density is $2.3 \mathrm{~g} / \mathrm{cm}^{3}$, which is the density at the axis. The shock front has moved further away from the axis, and the density of the shocked material is $9.94 \mathrm{~g} / \mathrm{cm}^{3}$.

Figure 9(c) shows the density distribution at $1000 \mathrm{~ns}$, when 40 bunches have already deposited their energy in the target. It is seen from the color scale that the minimum value of density (at the axis) is $1.13 \mathrm{~g} / \mathrm{cm}^{3}$, while the shock front is at a radial position of $0.7 \mathrm{~cm}$.

In a similar manner, it is seen in Fig. 9(d) that the density at the axis has become $0.83 \mathrm{~g} / \mathrm{cm}^{3}$, which is less than $10 \%$ of the solid copper density. The density of the shock front is $10.13 \mathrm{~g} / \mathrm{cm}^{3}$, and the shock front has moved to a radial position of around $1 \mathrm{~cm}$. A significant extension of the lowdensity cavity in the longitudinal direction is also visible, which, in fact, is the result of the hydrodynamic tunneling phenomenon.
Figure 10(a) shows the density vs the axis at different times during the irradiation. It is seen from curve 1 that, at $t=250 \mathrm{~ns}$, the minimum density is about $3.5 \mathrm{~g} / \mathrm{cm}^{3}$. It is interesting to note that the minima of the density curve occur at the same position where the maxima of the corresponding energy-deposition curve lies, as seen in Fig. 3(a). This is because the higher energy deposition at this point produces a higher pressure [see the corresponding curve in Fig. 8(a)], which in turn generates stronger hydrodynamic effects that lead to a minimum density. It is also seen that the behavior of the density curve 1 reciprocates the behavior of the pressure curve 1 in Fig. 8(a). It is also interesting to note that the depletion front shown in Fig. 10(a) moves towards the right, which is again associated with the hydrodynamic tunneling.

Figure 10(b) presents the same parameters as Fig. 10(a) but at radial position $r=1 \mathrm{~mm}$. The profiles in the two figures show a similar qualitative behavior, although the density values shown in the curves of Fig. 10(b) are significantly higher than those in the corresponding curves of Fig. 10(a). It is also interesting to note that the rightward propagation of the density depletion front is slower in the case of Fig. 10(b) in comparison with Fig. 10(a). 


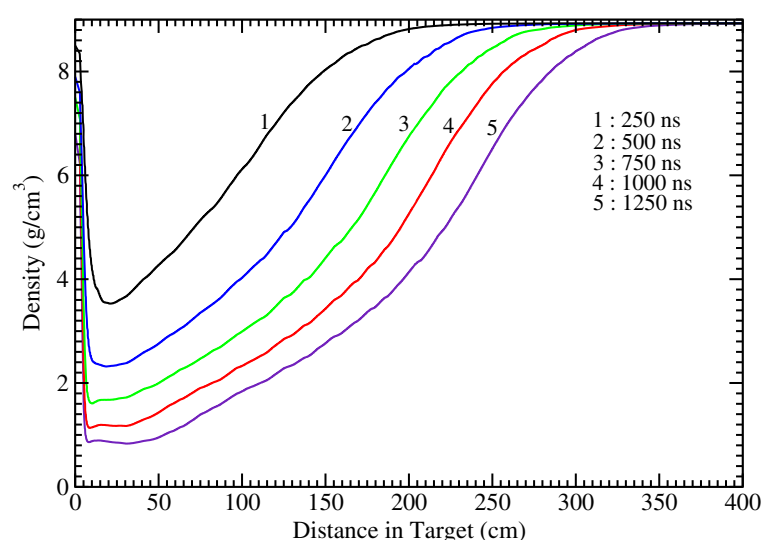

(a)

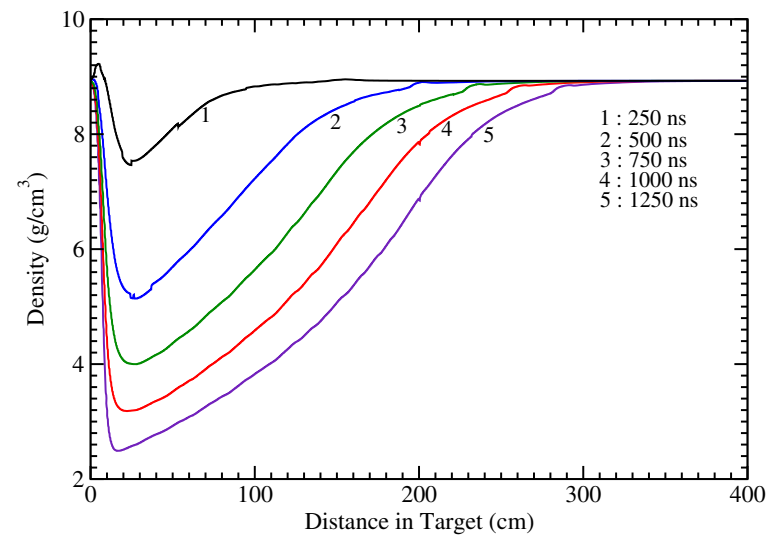

(b)

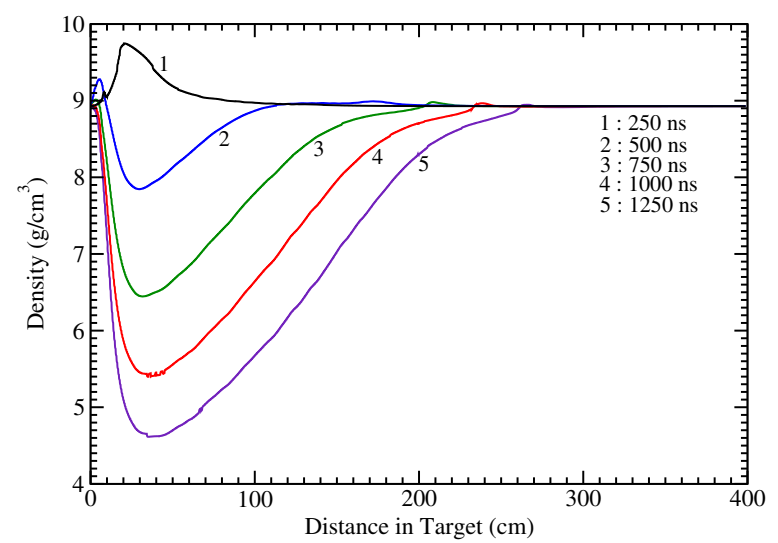

(c)

FIG. 10. Density profiles vs target cylinder at different times: (a) along the axis at $r=0$, (b) at $r=1 \mathrm{~mm}$, and (c) at $r=2 \mathrm{~mm}$.

Figure 10(c) shows the density vs the length at different times at a radial position of $2 \mathrm{~mm}$. Curve 1 shows that, at $t=250 \mathrm{~ns}$, the material density has increased to about $9.8 \mathrm{~g} / \mathrm{cm}^{3}$, which is contrary to the behavior of the corresponding curves in the other two figures. This is because, at this time, the shock front arrives at the radial position of $2 \mathrm{~mm}$ that compresses the material, thereby generating a supersolid density. The next curve shows that, at $t=500 \mathrm{~ns}$, the density has been reduced, because the shock front has moved further outwards along the radius.
The remaining curves show further density reduction and continuous rightward propagation of the density depletion front.

The above figures show the strong density depletion due to the radial shock wave and the resulting hydrodynamic tunneling of the protons and the shower.

\section{Target physical state}

It is important to know how the physical state of the target material will evolve during the process of irradiation. For this purpose, we present in Figs. 11(a)-11(d) the state of the target material at four different times. This information has been obtained from the semiempirical EOS data $[36,37]$ that are included in the BIG2 code.

Figure 11(a) has been plotted at $t=250 \mathrm{~ns}$, which shows that the entire beam-heated region has been liquefied while the melting zone surrounding this liquid region is clearly seen.

Figure 11(b) shows that, at $t=500 \mathrm{~ns}$, the liquid region has been significantly extended and the melting front has further propagated, both in the radial as well as in the longitudinal direction. The extension of the liquefied zone in the longitudinal direction is due to the deeper penetration of the protons and the shower due to the hydrodynamic tunneling. In the radial direction, as more and more energy is deposited by the shower generated by the incoming proton bunches, the material melts when the melting threshold is achieved.

Figure 11(c) shows that, at $t=1000 \mathrm{~ns}$, a significant increase in the volume of the liquefied material has taken place. Moreover, a small pencil-shaped region of a gaseous or plasma phase has also appeared along the cylinder axis.

In Fig. 11(d), the physical state at $t=1250 \mathrm{~ns}$ is presented. It is seen that a substantial part of the target has been liquefied, and a noticeable gaseous or plasma zone also exists at and around the axis. The beam-heated part of the target is thus converted into different phases of HED matter, including melting, expanded as well as compressed hot liquid, gas, and weakly ionized plasma. This is also a very important field of research.

It is therefore clear that, in the case of an uncontrolled release of the beam energy, only a few bunches of the FCC can cause irreversible damage to the accelerator components and other equipment. This demands great care in the handling of these beams that store a very large amount of energy, and a secure and robust machine protection system must be designed.

\section{Estimation of beam penetration length considering hydrodynamic tunneling}

Because of the storage and time limitations of the computer, it is not currently possible to carry out simulations of the interaction of all 10600 FCC bunches with the target. Nevertheless, it is possible to have a very good estimate of the penetration length of the full FCC beam in 


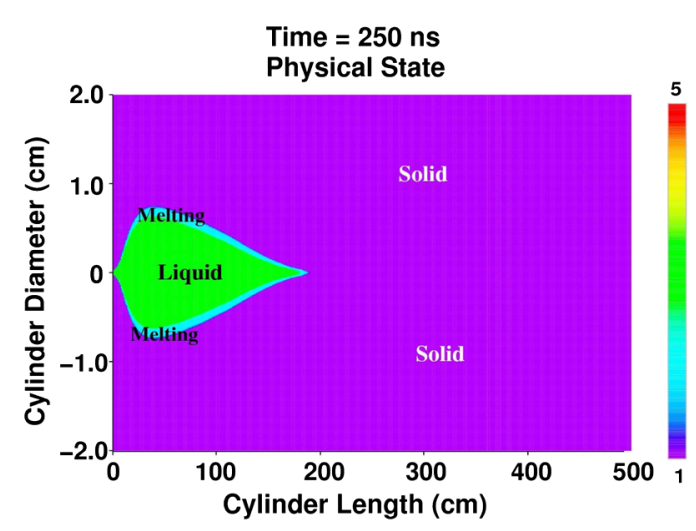

(a)

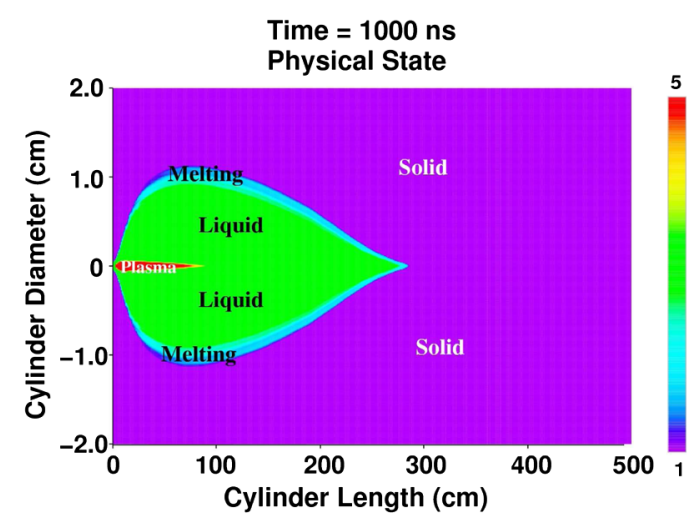

(c)

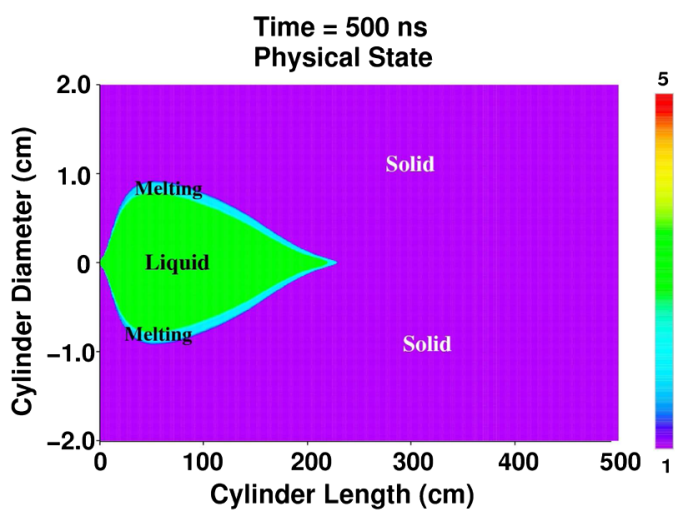

(b)

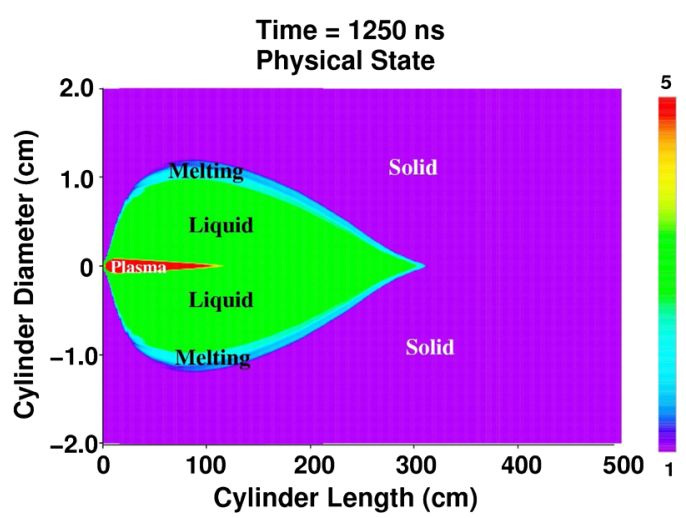

(d)

FIG. 11. Target physical state calculated by BIG2 (a) at $t=250 \mathrm{~ns}$ (10 bunches delivered), (b) at $t=500 \mathrm{~ns}$ (20 bunches delivered), (c) at $t=1000 \mathrm{~ns}$ (40 bunches delivered), and (d) at $t=1250 \mathrm{~ns}$ (50 bunches delivered).

the target material by analyzing our calculations that are done with a limited number of bunches. In fact, when the first FCC bunch deposits energy in a solid material, a huge pressure of about 1 Mbar is generated along the axis. This high pressure generates a very strong shock wave that leads to the rapid removal of the material from the axis, leading to fast density depletion. The second bunch, after passing through this low-density region, bores deeper into the solid part of the target, and the depletion front moves ahead. However, the pressure starts to drop in the low-density material that slows down the hydrodynamics, which in turn slows down the density reduction rate in the heated region. As a consequence, the speed of the depletion front slows with time until a steady state is achieved. The speed of the depletion front then becomes constant, and by multiplying this speed with the total length of the bunch train, one can estimate the penetration depth of the entire beam.

In Fig. 12, we present the density on the axis vs the target length at different times during irradiation. Curve " $a$ " represents the time when two bunches have been delivered, while later curves are plotted using an interval of $150 \mathrm{~ns}$. We consider $6 \mathrm{~g} / \mathrm{cm}^{3}$ as the reference point on the density curve and calculate the speed with which this point moves towards the right. At $t=800 \mathrm{~ns}$, the depletion front achieves a steady speed, as in each of the following
$150 \mathrm{~ns}$ time intervals it covers equal distances $x 1, x 2$, and $x 3$, respectively. In the simulations, $x 1=16.6 \mathrm{~cm}$, $x 2=16.4 \mathrm{~cm}$, and $x 3=16.8 \mathrm{~cm}$, which amounts to a total penetration length of $49.8 \mathrm{~cm}$ covered in $450 \mathrm{~ns}$. This leads to a steady average speed of $1.1 \times 10^{6} \mathrm{~m} / \mathrm{s}$. The total beam duration considering all 10600 bunches is $265 \mu$ s that leads

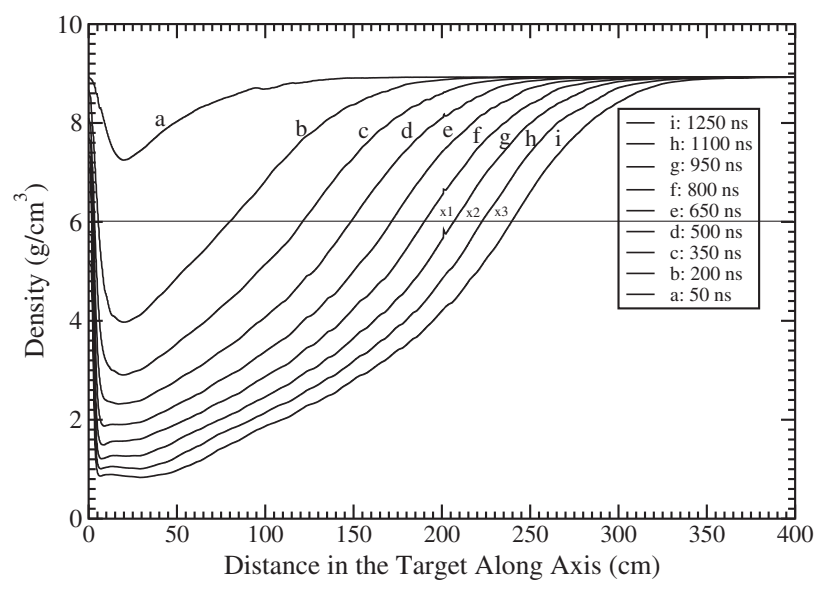

FIG. 12. Density on the axis vs distance in the target at different times, calculation of the penetration distance of the FCC beam in a solid copper target. 
to a penetration distance of about $290 \mathrm{~m}$. This means that in the case of a wrong deflection of the beam, the beam and the shower will penetrate through about $290 \mathrm{~m}$ of solid copper. If one considers a $50 \mathrm{TeV}$ proton beam, the penetration distance could be up to $350 \mathrm{~m}$.

\section{CONCLUSIONS}

The interaction of $40 \mathrm{TeV}$ proton bunches with a solid copper target has been analyzed employing an energydeposition code (FLUKA) and a two-dimensional hydrodynamic code (BIG2) iteratively. The purpose of this work is to estimate the damage caused by a wrong deflection of a FCC beam into accelerator components like magnets or other experimental equipment. A very important aspect of this problem is to estimate the penetration length of the entire FCC beam and the shower it generates, into different materials. Because of several limitations, for example, the required computing time, it is not currently possible to do these calculations considering all 10600 FCC bunches. Nevertheless, one can estimate the penetration length by doing the calculations using a smaller number of bunches until the penetration speed of the protons and the shower along the axis becomes constant. From this speed, it is possible to calculate the distance the protons will penetrate considering the entire beam duration. Our simulations, which have been done using only 50 bunches, show that the final speed with which the penetration wave will travel after achieving a steady state is about $1.1 \times 10^{6} \mathrm{~m} / \mathrm{s}$. This implies that the full $40 \mathrm{TeV}$ proton beam and the shower will penetrate around $290 \mathrm{~m}$ in solid copper. If one considers $50 \mathrm{TeV}$ protons, which is now the nominal FCC particle energy, this distance could be of the order of $350 \mathrm{~m}$.

It is also important to note that, although the transverse size of the beam is small with a standard deviation of only $0.2 \mathrm{~mm}$, it generates a very strong shower that deposits significant energy far beyond the effective radius of the particle beam. Simulations have shown that at $t=1250 \mathrm{~ns}$, when only 50 bunches have interacted with the target, the specific energy deposition at radial position $r=1 \mathrm{~cm}$ is about $0.65 \mathrm{~kJ} / \mathrm{g}$ that leads to a temperature of $1900 \mathrm{~K}$. Even at $r=2 \mathrm{~cm}$ (cylinder surface), the temperature has already increased to about $550 \mathrm{~K}$. It is therefore clear that, after the delivery of a certain number of bunches, enough energy will accumulate in the outer part of the target that will liquefy the material. This could cause serious damage of accelerator equipment and the machine protection systems must be designed to prevent such accidents.

[1] A. Ferrari, P. R. Sala, A. Fasso, and J. Ranft, CERN Technical Report No. CERN-2005-010.

[2] A. Fasso, A. Ferrari, S. Roesler, R. P. Sala, G. Battistoni, F. Cerutti, E. Gadioli, M. V. Garzelli, F. Ballarini, A.
Ottolenghi, A. Empl, and J. Ranft, in Proceedings of the Conference on Computing in High Energy and Nuclear Physics, La Jolla, 2003.

[3] V. E. Fortov, B. Goel, C. D. Munz, A. L. Ni, A. Shutov, and O. V. Vorobiev, Numerical simulations of nonstationary fronts and interfaces by the Godunov method in moving grids, Nucl. Sci. Eng. 123, 169 (1996).

[4] N. A. Tahir, B. Goddard, V. Kain, R. Schmidt, A. Shutov, I. V. Lomonosov, A. R. Piriz, M. Temporal, D. H. H. Hoffmann, and V.E. Fortov, Impact of 7-Tev/c large hadron collider proton beam on a copper target, J. Appl. Phys. 97, 083532 (2005).

[5] N. A. Tahir, V. Kain, R. Schmidt, A. Shutov, I. V. Lomonosov, V. Gryaznov, A. R. Piriz, M. Temporal, D. H. H. Hoffmann, and V.E. Forotv, The CERN Large Hadron Collider as a Tool to Study High-Energy Density Matter, Phys. Rev. Lett. 94, 135004 (2005).

[6] N. A. Tahir, R. Schmidt, A. Shutov, I. V. Lomonosov, A. R. Piriz, D. H. H. Hoffmann, C. Deutsch, and V. E. Fortov, Large Hadron Collider at CERN: Beams generating highenergy-density matter, Phys. Rev. E 79, 046410 (2009).

[7] N. A. Tahir, J. Blanco Sancho, A. Shutov, R. Schmidt, and A. R. Piriz, Impact of high energy high intensity proton beams on targets: Case studies for Super Proton Synchrotron and Large Hadron Collider, Phys. Rev. ST Accel. Beams 15, 051003 (2012).

[8] R. Schmidt, J. Blanco Sancho, F. Burkart, D. Grenier, D. Wollmann, N. A. Tahir, A. Shutov, and A. R. Piriz, First experimental evidence of hydrodynamic tunneling of ultrarelativistic protons in extended solid copper target at the CERN HiRadMat facility, Phys. Plasmas 21, 080701 (2014).

[9] F. Burkart, R. Schmidt, V. Raginel, D. Wollmann, N. A. Tahir, A. Shutov, and A. R. Piriz, Analysis of $440 \mathrm{GeV}$ proton beam-matter interaction experiments at the High Radiation Materials test facility at CERN, J. Appl. Phys. 118, 055902 (2015).

[10] N. A. Tahir, F. Burkart, A. Shutov, R. Schmidt, D. Wollmann, and A. R. Piriz, Simulations of beam-matter interaction experiments at the CERN HiRadMat facility and prospects of high-energy-density physics research, Phys. Rev. E 90, 063112 (2014).

[11] R. O. Bangerter, J. W. K. Mark, and A. R. Thiessen, Heavy ion inertial fusion: Initial survey of target gain versus ionbeam parameters, Phys. Lett. A 88, 225 (1982).

[12] C. Deutsch, Inertial confinement fusion driven by intense ion beams, Ann. Phys. (Paris) 11, 1 (1986).

[13] N. A. Tahir and K. A. Long, Numerical simulation and theoretical analysis of implosion, ignition and burn of heavy-ion-beam reactor-size ICF targets, Nucl. Fusion 23, 887 (1983).

[14] N. A. Tahir and K. A. Long, Numerical modelling of radiation Marshak Waves, Laser Part. Beams 2, 371 (1984).

[15] J. J. Barnard et al., Theory and simulation of warm dense matter targets, Nucl. Instrum. Methods Phys. Res., Sect. B 577, 275 (2007).

[16] N. A. Tahir, D. H. H. Hoffmann, J. A. Maruhn, K.-J. Lutz, and R. Bock, Numerical simulations and theoretical analysis of proposed heavy-ion-matter experiments at 
the GSI Darmstadt accelerator facility, Phys. Plasmas 5, 4426 (1998).

[17] N. A. Tahir, D. H. H. Hoffmann, A. Kozyreva, A. Tauschwitz, A. Shutov, J. A. Maruhn, P. Spiller, U. Neuner, J. Jacoby, M. Roth, R. Bock, H. Juranek, and R. Redmer, Metallization of hydrogen using heavy-ion-beam implosion of multilayered cylindrical targets, Phys. Rev. E 63, 016402 (2001).

[18] N. A. Tahir, C. Deutsch, V. E. Fortov, V. Gryaznov, D. H. H. Hoffmann, M. Kulish, I. V. Lomonosov, V. Mintsev, P. Ni, D. Nikolaev, A. R. Piriz, N. Shilkin, P. Spiller, A. Shutov, M. Temporal, V. Ternovoi, S. Udrea, and D. Varentsov, Proposal for the Study of Thermophysical Properties of High-Energy-Density Matter Using Current and Future Heavy-Ion Accelerator Facilities at GSI Darmstadt, Phys. Rev. Lett. 95, 035001 (2005).

[19] N. A. Tahir et al., Ultrahigh compression of water using intense heavy ion beams: laboratory planetary physics, New J. Phys. 12, 073022 (2010).

[20] N. A. Tahir, A. Shutov, A. P. Zharkov, A. R. Piriz, and Th. Stöhlker, Generation of plane shocks using intense heavy ion beams: Application to Richtmyer-Meshkov instability growth studies, Phys. Plasmas 18, 032704 (2011).

[21] N. A. Tahir, P. Spiller, A. Shutov, I. V. Lomonosov, V. Gryaznov, A. R. Piriz, G. Wouchuk, C. Deutsch, V. E. Fortov, D. H. H. Hoffmann, and R. Schmidt, HEDgeHOB: High-energy density matter generated by heavy ion beams at the future facility for antiprotons and ion research, Nucl. Instrum. Methods Phys. Res., Sect. A 577, 238 (2007).

[22] A. R. Piriz, O. D. Cortazar, J. J. Lopez Cela, and N. A. Tahir, The Rayleigh-Taylor instability, Am. J. Phys. 74, 1095 (2006).

[23] A. R. Piriz, J. J. Lopez Cela, N. A. Tahir, and D. H. H. Hoffmann, Richtmyer-Meshkov instability in elasticplastic media, Phys. Rev. E 78, 056401 (2008).

[24] A. R. Piriz, J. J. Lopez Cela, and N. A. Tahir, Linear analysis of incompressible Rayleigh-Taylor instability in solids, Phys. Rev. E 80, 046305 (2009).

[25] U. N. Funk, R. Bock, M. Dornik, M. Geissel, M. Stetter, S. Stöwe, N. A. Tahir, and D. H. H. Hoffmann, High energy density in solid rare gas targets and solid hydrogen, Nucl. Instrum. Methods Phys. Res., Sect. A 415, 68 (1998).

[26] H. Geissel et al., The Super-FRS project at GSI, Nucl. Instrum. Methods Phys. Res., Sect. B 204, 71 (2003).
[27] N. A. Tahir, V. Kim, A. Matveichev, A. V. Ostrik, A. V. Shutov, I. V. Lomonosov, A. R. Piriz, J. J. Lopez Cela, and D. H. H. Hoffmann, High energy density and beam induced stress related issues in solid graphite Super-FRS fast extraction targets, Laser Part. Beams 26, 273 (2008).

[28] N. A. Tahir, H. Weick, A. Shutov, V. Kim, A. Matveichev, A. Ostrik, V. Sultanov, I. V. Lomonosov, A. R. Piriz, J. J. Lopez Cela, and D. H. H. Hoffmann, Simulations of solid graphite target for high intensity fast extracted uranium beams for the Super-FRS, Laser Part. Beams 26, 411 (2008).

[29] N. A. Tahir, A. Matveichev, V. Kim, A. Ostrik, A. Shutov, V. Sultanov, I. V. Lomonosov, A. R. Piriz, and D. H. H. Hoffmann, Three-dimensional simulations of a solid graphite target for high intensity fast extracted uranium beams for the Super-FRS, Laser Part. Beams 27, 9 (2009).

[30] N. A. Tahir, V. Kim, E. Lamour, I. V. Lomonosov, A. R. Piriz, J. P. Rozet, Th. Stöhlker, V. Sultanov, and D. Vernhet, Two-dimensional thermal simulations of an aluminum beam stripper for experiments at SPIRAL2, Nucl. Instrum. Methods Phys. Res., Sect. B 276, 66 (2012).

[31] N. A. Tahir, V. Kim, E. Lamour, I. V. Lomonosov, A. R. Piriz, J. P. Rozet, Th. Stöhlker, V. Sultanov, and D. Vernhet, Two-dimensional thermal simulations of aluminum and carbon ion strippers for experiments at SPIRAL2 using the highest beam intensities, Nucl. Instrum. Methods Phys. Res., Sect. B 290, 43 (2012).

[32] N. A. Tahir, V. Kim, B. Scklitt, W. Barth, L. Groening, I. V. Lomonosov, A. R. Piriz, T. Stöhlker, and H. Vormann, Phys. Rev. Accel. Beams 17, 041003 (2014).

[33] D. C. Wilson, C. A. Wingate, J. C. Goldstein, R. P. Godwin, and N. V. Mokhov, in Proceedings of the 15th Particle Accelerator Conference, PAC-1993, Washington, DC, 1993 (IEEE, New York, 1993), pp. 3090-3092.

[34] N. V. Mokhov and F. Cerutti, Report No. CERN-2016-002.

[35] J. F. Ziegler, J. P. Biersack, and U. Littmark, The Stopping and Range of Ions in Matter (Pergamon Press, New York, 1966).

[36] I. V. Lomonosov, Multi-phase equation of state for aluminum, Laser Part. Beams 25, 567 (2007).

[37] I. V. Lomonosov and N. A. Tahir, Theoretical investigation of shock wave stability in metals, Appl. Phys. Lett. 92, 101905 (2008). 